\title{
SMA observations of massive star forming regions
}

\author{
Sheng-Yuan Liu ${ }^{1}$ and the SMA Team ${ }^{1,2}$ \\ ${ }^{1}$ Academia Sinica Institute of Astronomy and Astrophysics, \\ P.O. Box 23-141,Taipei 106, Taiwan, R.O.C. \\ email: syliu@asiaa.sinica.edu.tw \\ ${ }^{2}$ Harvard-Smithsonian Center for Astrophysics, \\ 60 Garden Street, Cambridge, MA 02138, U.S.A.
}

\begin{abstract}
The Submillimeter Array located atop of Mauna Kea in Hawaii is a collaborative project of the Smithsonian Astrophysical Observatory and the Academia Sinica Institute of Astronomy and Astrophysics. The high angular resolution provided by the SMA is particularly suitable for studying massive star-forming cores which often exhibit strong (sub)millimeter continuum and spectral features but mostly locate within crowded regions at large distances. We report the latest SMA status and recent results in the area of massive star formation obtained with the array.
\end{abstract}

Keywords. interferometers; high angular resolution; HII regions; jets and outflows; molecules

\section{Introduction}

The Submillimeter Array (SMA) is a collaborative project of the Smithsonian Astrophysical Observatory (SAO) and the Academia Sinica Institute of Astronomy and Astrophysics (ASIAA). With its initial concept conceived at the SAO in 1983, the project identified Mauna Kea as the observatory site and the construction started in the early 1990s. In 1996, ASIAA joined the project by expending the original six-element array into an eight-element array, nearly doubling the imaging speed. By the end of 2003, all eight elements of the SMA were deployed on top of Mauna Kea. The SMA was formally dedicated on 2003 November 22, by which time regular operation phase started. Table 1 gives a list of basic characteristics of the array.

In studying massive star formation, many key questions remain to be answered. For example, what are the initial conditions/environments within which massive stars form?

Table 1. The Basic Characteristics of the SMA

\begin{tabular}{|c|c|}
\hline Antennas & $\begin{array}{l}\text { Eight reflectors, } 6 \text { meters in diameter } \\
\text { Carbon Fiber backup structure, Machined cast aluminum panels } \\
\text { Primary reflector f } / 0.4 \text {, surface } 12 \mu \mathrm{m} \text { rms, Chopping secondary reflector }\end{array}$ \\
\hline Configurations & 4 rings, 24 pads, up to 8 antennas per ring \\
\hline Baselines & 8 to 508 meters (15-250 meters with current pads) \\
\hline Frequencies & 180 to $900 \mathrm{GHz}$ (current bands: $175-255,250-350,600-720 \mathrm{GHz}$ ) \\
\hline Receivers & up to 8 per cryostat (simultaneous dual bands/polarizations) \\
\hline Correlator & $\begin{array}{l}\text { Flexible Hybrid analog-digital design } \\
\text { up to } 2 \mathrm{GHz} \text { bandwidths, } 2 \text { receivers, } 8 \text { stations }\end{array}$ \\
\hline Filed of View & 70 to 14 arcsec \\
\hline Resolution & 0.5 to 0.1 arcsec \\
\hline Sensitivity & for $1 \mathrm{~mm} \mathrm{H} 2 \mathrm{O}, 8 \mathrm{hr}, 2 \mathrm{GHz}$ bandwidth: $\Delta \mathrm{S} \sim 0.4,1,10 \mathrm{mJy}$ at 230,345 , and $690 \mathrm{GHz}$ \\
\hline
\end{tabular}


How general are the outflow and disk phenomena associated with young stellar objects toward the massive end of their mass spectrum? Where and how these outflows get launched and regulated? How these disks might evolve? Given their rapid evolution, young massive stars are likely to be deeply emdeded in the natal clouds. Dust surrounding these objects thus absorbs almost all stellar radiation and reradiates in the far-infrared and submillimeter wavelengths, while the surrounding dense and warm molecular gas also exhibits intense submillimeter line emission. The SMA therefore provides the crucial resolving power in particular for studying massive star-forming cores which mostly locate within crowded regions at large distances. We report results from observations obtained with the SMA in the field of massive star formation during its early commissioning phase as well as the recent regular operation seasons.

\section{Recent Results}

\subsection{G5.89-0.39}

G5.89-0.39 is a shell-like ultracompact (UC) HII region (Wood \& Churchwell 1989) at a distance of $\sim 2 \mathrm{kpc}$ associated with a molecular outflow (e.g. Acord et al. 1997). Sollins et al. (2004) mapped G5.89-0.39 and its surroundings with the SMA at 2.8" $\times 1.8$ " angular resolution in $1.3 \mathrm{~mm}$ continuum, $\mathrm{SiO} 5-4$ and eight other molecular lines. The molecular outflow is resolved for the first time and is definitely bipolar. The $\mathrm{SiO} 5-4$ positionvelocity structure with emission exceeding $30 \mathrm{~km} \mathrm{~s}^{-1}$ suggests the outflow is mainly in the plane of the sky and highly energetic. The $1.3 \mathrm{~mm}$ continuum is likely the outflow driving source as its peak lies on the projected outflow axis, halfway between the peaks of the two outflow lobes. In addition, the slightly extended continuum emission seems to show a physical connection to both outflow lobes in the form of extensions along the outflow axis.

\subsection{IRAS 18089-1732}

IRAS 18089-1732, at a distance of $\sim 3.6 \mathrm{kpc}$ with $10^{4.5} \mathrm{~L}_{\odot}$ in luminosity, is one of the young high-mass protostellar object (HMPO) among the sample of 69 HMPOs selected and surveyed by Sridharan et al. (2002). Past observations revealed a massive core greater than $2000 \mathrm{M}_{\odot}$ in millimeter continuum associated with maser emission and weak $3.6 \mathrm{~cm}$ radio continuum (Beuther et al. 2002b, 2002d). Observations with the SMA at 217 and $344 \mathrm{GHz}$ by Beuther et al. 2004a, 2004b detected a total of 34 molecular lines from 16 molecules/isotopomers, supporting the hot-core nature of this object. The large number of observed $\mathrm{HCOOCH}_{3}$ lines allowed an estimate of the temperature for the central region to be $350 \pm 100 \mathrm{~K}$ (Beuther et al. 2004a). The SiO 5-4 data show a collimated outflow in the northern direction. In contrast, the $\mathrm{HCOOCH}_{3}$ 20-19 line, which traces high-density gas, is confined to the very center of the region and shows a velocity gradient across the core, most likely originating from a rotating disk that is influenced by outflows and infall (beuther04b. The combined evidence supports the scenario in which high-mass stars form via disk accretion accompanies by collimated jets/outflows, just in a similar fashion as their low-mass counterparts.

\subsection{IRAS 20126+4104}

IRAS $20126+4104$ is the first high-mass protostellar object identified with a jet and a disk. The $\mathrm{SiO}$ and $\mathrm{HCO}^{+}$emission has been observed tracing a well collimated bipolar jet/outflow located symmetrically around the central YSO (Cesaroni et al. 1997, 1999). Images in the highly excited CO 7-6 line also exhibit two hot spots near the tip of the SiO jet (Kawamura et al. 1999). The hot spots, separated by about 20", coincide with the H2 

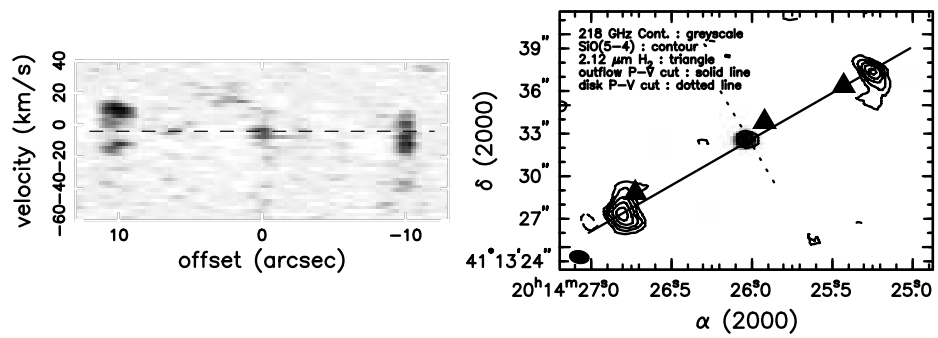

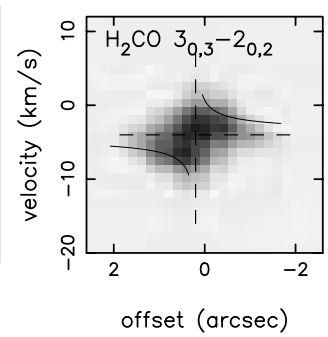

Figure 1. Left panel: position-velocity diagram of $\mathrm{SiO} 5-4$ emission along the jet/outflow. Middle panel: $1.3 \mathrm{~mm}$ continuum and integrated $\mathrm{SiO}$ 5-4 emission of IRAS 20126+4104. Right panel: position-velocity diagram of $\mathrm{H}_{2} \mathrm{CO} 3_{0,3}-2_{0,2}$ emission along the disk.

knots (Shepherd et al. 2000). Perpendicular to the jet/outflow, both the $\mathrm{CH}_{3} \mathrm{CN}$ as well as the $\mathrm{NH}_{3}(1,1)$ and $(2,2)$ lines show an elongated disk-like structure with its velocity field consistent with Keplerian rotation (Cesaroni et al. 1997, 1999; Zhang, Hunter, \& Sridharan 1998). Imaged with the SMA by Liu et al. the integrated $\mathrm{SiO} 5$-4 emission at $217 \mathrm{GHz}$ depicts a clear bow-shock morphology coinciding with the $\mathrm{H}_{2}$ emission. The emission location suggests $\mathrm{SiO}$ is mostly concentrated/excited immediately behind the bow shock apex. The "tail" morphology appears extending in the direction of the jet precession. Along the disk direction, the position-velocity diagrams of the $K=0$ and $K=2$ $\mathrm{H}_{2} \mathrm{CO}$ lines at $218 \mathrm{GHz}$ suggest spin-up motions, most likely associated with Keplerian rotation. Moreover, a rich chemistry exists in both the core/disk and the outflow - in addition to $\mathrm{H}_{2} \mathrm{CO}, \mathrm{CH}_{3} \mathrm{OH}, \mathrm{DCN}$, and $\mathrm{HC}_{3} \mathrm{~N}$ were all detected toward the central YSO. In the outflow, while $\mathrm{SiO}$ emission appears to be stronger toward the $\mathrm{SE}$ knot, little $\mathrm{H}_{2} \mathrm{CO}$ or $\mathrm{CH}_{3} \mathrm{OH}$ emission was detected. In contrast, $\mathrm{SiO}, \mathrm{H}_{2} \mathrm{CO}$, and $\mathrm{CH}_{3} \mathrm{OH}$ were detected toward the NW knot with their peaks progressively located away from the bow shock tip.

\subsection{Cepheus A-East}

Within a molecular complex associated with Cepheus OB3 some 725 pc away, the Cepheus A region displays various star formation signatures (Torrelles et al. 1993). For example, multiple outflows on different scales have been found (Rodríguez et al. 1980; Ho, Moran, \& Rodríguez 1982; Hayashi, Hasegawa, \& Kaifu 1988). In Cepheus A East, no less than sixteen radio-continuum sources were detected (Hughes \& Wouterloot 1984; Garay et al. 1996). Among these, HW2 is suggested to be the driving source for the molecular outflows. Using the SMA at $343 \mathrm{GHz}$ with an angular resolution of $\sim 1.5 "$, Brogan et al. (private communication) detected submillimeter counterparts of the radio sources HW2, HW3b, and HW3c. Numerous lines from hot core tracing species, such as $\mathrm{CH}_{3} \mathrm{OH}, \mathrm{CH}_{2} \mathrm{CO}$, $\mathrm{HCOOCH}_{3}, \mathrm{CH}_{3} \mathrm{OCH}_{3}$, and $\mathrm{C}_{2} \mathrm{H}_{5} \mathrm{CN}$ are all detected toward the $\mathrm{HW} 2$ core.

\subsection{IRAS $05358+3543$}

IRAS $05358+3543$, at a distance of $1.8 \mathrm{kpc}$, is another massive star forming object among the $69 \mathrm{HMPO}$ sample studied by Sridharan et al. (2002). Both $\mathrm{H}_{2} \mathrm{O}$ and $\mathrm{CH}_{3} \mathrm{OH}$ masers have been detected in this region (Tofani et al. 1995; Minier et al. 2000). Similar to Cepheus A East, multiple outflows have also been imaged in CO (Beuther et al.2002a). The SMA observations carried out by Beuther et al. (private communication) successfully imaged dust condensations in this region at $338 \mathrm{GHz}$ as well as line emission from various complex molecules. The large number of detected $\mathrm{CH}_{3} \mathrm{OH}$ lines at $348 \mathrm{GHz}$ are used for temperature and density analysis (see Leurini et al. 2005 in this volume). 


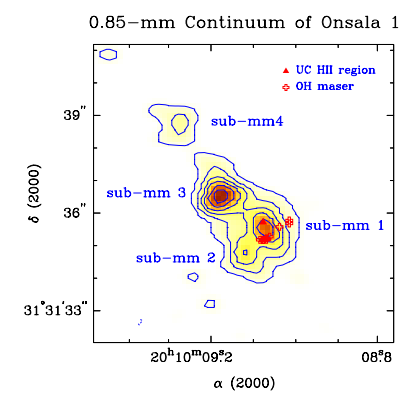

Figure 2. $850 \mu \mathrm{m}$ continuum of Onsala 1 shown in both contours and grayscale.

\subsection{Onsala 1}

Given its compactness, Onsala 1 is one of the smallest, hence possibly the youngest UCHII regions in the Galaxy. Located at a distance of $1.8 \mathrm{kpc}$, its physical size would be around $0.01 \mathrm{pc}$, implying a very young age of 1000 years. $\mathrm{H}_{2} \mathrm{O}$ and $\mathrm{OH}$ maser emission, has also been found in this region (Downes et al. 1979; Ho et al. 1983). At an angular resolution of 0.6 ", recent $345 \mathrm{GHz}$ SMA observations by Su et al. (private communication) revealed at least four components (denoted as sub-mm 1-4) within a 5" field (i.e., $0.05 \mathrm{pc}$ ). Sub$\mathrm{mm} 1$ appears associated with the UC HII region and $\mathrm{OH}$ masers and is dominated by free-free emission from ionized gas as recent VLA continuum observations at 3.6 and $1.3 \mathrm{~cm}$ suggested. In contrast, the newly identified sub-mm 2, 3, and 4 appear to be dominated by dust emission with no radio counterpart. Furthermore, sub-mm $2 \& 3$ are spatially coincident with $\mathrm{H}_{2} \mathrm{O}$ maser spots (Zheng et al. 1985), indicating both are likely hot core candidates, which represent the sites of high-mass star formation prior to the UC HII region phase. The results strongly suggest that Onsala 1 harbors massive stars at different but extremely young evolutionary stages in their formation.

\subsection{Orion $K L$}

At a distance of $450 \mathrm{pc}$, Orion KL is the nearest and most studied region of massive star formation. The region exhibits a complex cluster of near- to mid-infrared sources (e.g. Dougados et al. 1993; Greenhill et al. 2004). At least two outflows at different scales are observed originating from this region: one large scale high-velocity outflow in the southeast-northwest direction (e.g., Allen \& Burton 1993; Wright et al. 1995; Schultz et al. 1999) and at a smaller scale one lower velocity outflow in the northeast-southwest direction (e.g., Genzel \& Stutzki 1989; Blake et al. 1996; Chrysostomou et al. 1997; Stolovy et al. 1998). The driving source(s) of the outflows are, however, uncertain. Possible candidates are the radio sources I and/or the infrared source n, also known as radio source L (Menten \& Reid 1995).

SMA observations by Beuther et al. (2004c, 2005) at 338 and $348 \mathrm{GHz}$ detected about 145 spectral lines from 25 species/isotopologues at different vibrationally excited states. Chemical differentiation within the region is evident as reported by past studies: most nitrogen bearing molecules are strong toward the hot core, whereas oxygen-bearing molecules peak toward the south-west in the so-called compact ridge. The $\mathrm{SiO}$ lines, in particular, are found to trace the collimated low-velocity molecular jet emanating from source I as well as larger-scale emission likely associated with a different outflow.

The $850 \mu \mathrm{m}$ continuum image resolved source I from the hot core and detected, for the first time, source $\mathrm{n}$ at a wavelength shorter than $7 \mathrm{~mm}$. Furthermore, a new continuum peak between the sources I and n, SMA1, is also found. The radio source I lies close to the center of a biconical outflow traced by $\mathrm{SiO}$ and $\mathrm{H}_{2} \mathrm{O}$ maser emission (Gezari 1992; 

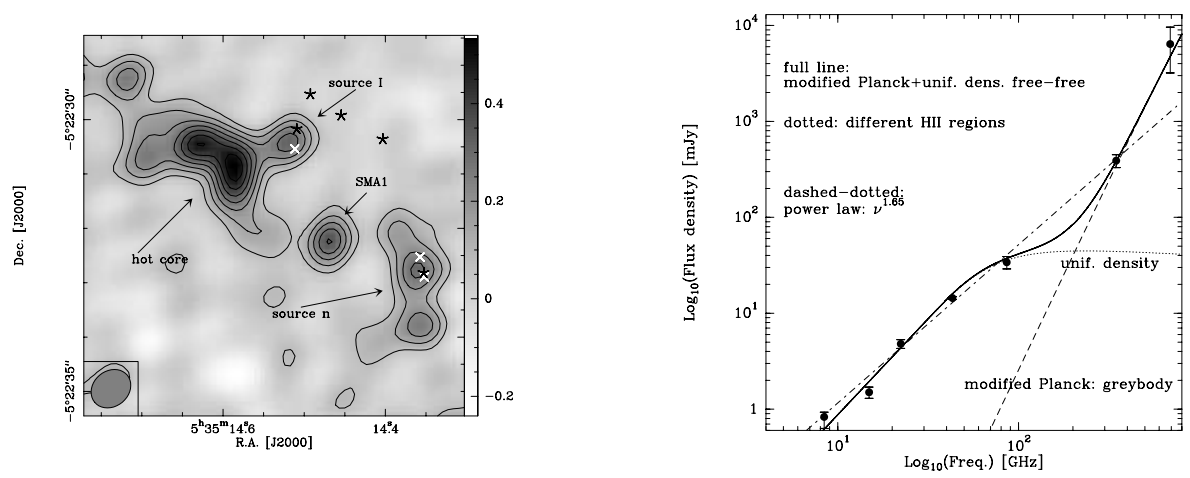

Figure 3. Left Panels: submillimeter continuum of Orion KL at $850 \mu \mathrm{m}$ shown in both contours and grayscale. Right panel: The SED from 8 to $690 \mathrm{GHz}$ for source I in Orion KL (From H. Beuther, private communication)

Menten \& Reid 1995; Greenhill et al. 2004). Its spectral energy distribution (SED) from 8 to $86 \mathrm{GHz}$ can be explained by optically thick free-free emission. With the new measurement, the SED for source I from 8 to $348 \mathrm{GHz}$ allows two equally possible interpretations. One possibility is to fit the lower frequency part of the spectrum with proton-electron free-free emission, but to allow dust emission to dominate the submillimeter continuum. Alternatively, one can also fit a power law $\mathrm{S} \propto \nu^{\alpha}$ to the SED with $\alpha \sim 1.65 \pm 0.2$. This is similar to the spectral index observed toward Mira variable stars (Reid \& Menten 1997). Supporting evidence that the radio continuum forms under Mira-like conditions in a region with a temperature $\sim 1600 \mathrm{~K}$ and a density of $10^{11}-10^{12} \mathrm{~cm}^{-3}$ comes from the detection of $\mathrm{SiO}$ masers from source I.

The highlight comes from observations toward Orion KL at 680 and $690 \mathrm{GHz}$ during a recent high frequency band champaign. Through visibility fitting, a constraint on the source I flux density at $440 \mu \mathrm{m}$ of $6.4 \pm 3.2 \mathrm{Jy}$ is derived. The combined SED from 8 to 690 $\mathrm{GHz}$ clearly discriminates between the two models mentioned above and identifies source I as a deeply embedded protostellar object with optical thick free-free emission up to 100 $\mathrm{GHz}$ plus dust emission accounting for most of its submm flux. The $650 \mathrm{GHz}$ observations also confirmed SMA1 to be an independent protostellar source rather than an extension of the hot core. Finally, 24 spectral line features are observed in the bandpass, with a large fraction of lines undetected in previous single-element line survey (Schilke et al. 2001). Molecular emission from highly excited lines such as $\mathrm{CH}_{3} \mathrm{CN} 37_{2}-36_{2}, \mathrm{CH}_{3} \mathrm{OH}$ $22_{1}-21_{2}$, and $\mathrm{SO}_{2} 35_{3,33}-34_{2,32}$ all shows peaks toward source I, SMA1, and the hot core location, indicating dense and warm gas surrounding these objects.

\section{Future Prospects}

As regular science programs carry on, instrumental upgrades will continue to realize SMA's full capability. Among many items, efforts in the near future include the installation of receivers for simultaneous dual polarization capability, the link-up with the James Clerk Maxwell Telescope and the Caltech Submillimeter Observatory for sensitivity improvement, as well as the development of the remaining receiver bands for a complete frequency coverage.

\section{Acknowledgements}

The authors thank H. Beuther, Y.-N. Su, C. L. Brogan, and P. K. Sollins for discussing their results and providing the images in this paper. We also thank the whole SMA 
team from both SAO and IAA for their dedicated and continuous work in making this instrument possible.

\section{References}

Acord, J.M., Walmsley, C.M., \& Churchwell, E., 1997 ApJ, 475, 693

Allen, D.A., \& Burton, M.G., 1993 Nature, 363, 54

Beuther, H., Schilke, P., Gueth, F., McCaughrean, M., Andersen, M., Sridharan, T.K., \& Menten, K.M., 2002a $A \mathscr{E} A$, 387, 931

Beuther, H., Schilke, P., Menten, K.M., Motte, F., Sridharan, T.K., \& Wyrowski, F., 2002b ApJ, 566, 945

Beuther, H., Schilke, P., Sridharan, T.K., Menten, K.M., Walmsley, C.M., \& Wyrowski, F., 2002c $A \& A, 383,892$

Beuther, H., Walsh, A, Schilke, P., Sridharan, T.K., Menten, K.M., \& Wyrowski, F., 2002d $A \mathscr{G} A, 390,289$

Beuther, H., Zhang, Q., Hunter, T.R. et al., 2004a ApJL, 616, 19

Beuther, H., Hunter, T.R., Zhang, Q. et al., 2004b ApJL, 616, 23

Beuther, H. Zhang, Q., Greenhill, L.J. et al., 2004c ApJL, 616, 31

Beuther, H. Zhang, Q., Greenhill, L.J. et al., 2005 ApJL, accepted

Blake, G.A., Mundy, L.G., Carlstrom, J. E., et al., 1996 ApJL, 472, 49

Cesaroni, R., Felli, M., Testi, L., Walmsley, C.M. \& Olmi, L., 1997 A\& A, 325, 725

Cesaroni, R., Felli, M., Jenness, T., Neri, R., Robberto, M., Testi, L., \& Walmsley, C.M., 1999 $A \mathscr{E} A, 345,949$

Chrysostomou, A., Burton, M.G., Axon, D.J., et al., 1997 MNRAS, 289, 605

Dougados, C., Lena, P., Ridgway, S.T., Christou, J.C., \& Probst, R. G., 1993 ApJ, 406, 112

Downes, D., Genzel, R., Moran, J.M., Johnston, K.J., Matveenko, L.I., Kogan, L.R., Kostenko, V.I., \& Ronnang, B., 1979 A\&A, 79, 233

Garay, G., Ramírez, S., Rodríguez, L.F., Curiel, S., \& Torrelles, J.M., 1996 ApJ, 459, 193

Genzel, R., \& Stutzki, J., 1989 ARA\&A, 27, 41

Gezari, D.Y., 1992 ApJL, 396, 43

Greenhill, L. J., et al. 2004 ApJL, 605, 57

Hayashi, S.S., Hasegawa, T., \& Kaifu, N., 1988 ApJ, 332, 354

Ho, P.T.P., Moran, J.M., \& Rodríguez, L.F., 1982 ApJ, 262, 619

Ho, P.T.P., Vogel, S.N., Wright, M.C.H., \& Haschick, A.D., 1983 ApJ, 265, 295

Hughes, V.A., \& Wouterloot, J.G.A., 1984 ApJ, 276, 204

Kawamura, J.H., Hunter, T.R., Tong, C.-Y. et al., 1999 PASP, 111, 1088

Leurini, S., Schilke, P., Beuther, H., \& Menten, K.M., 2005 this volumn

Menten, K.M. \& Reid, M.J., 1995 ApJL, 445, 157

Minier, V., Booth, R.S., \& Conway, J.E., 2000 A\&A, 362, 1093

Reid, M.J., \& Menten, K.M., 1997 ApJ, 476, 327

Rodríguez, L.F., Ho, P.T.P., \& Moran, J.M., 1980 ApJL, 240, 149

Schilke, P., Benford, D.J., Hunter, T.R., Lis, D.C., \& Phillips, T.G., 2001 ApJS, 132, 281

Schultz, A.S.B., et al., 1999 ApJ, 511, 282

Shepherd, D.S., Yu, K.C., Bally, J., \& Testi, L., 2000 ApJ, 535, 833

Sollins P.K., Hunter, T.R., Battat, J. et al., 2004 ApJL, 616, 35

Sridharan, T.K., Beuther, H., Schilke, P., Menten, K.M., \& Wyrowski, F., 2002 ApJ, 566, 931

Stolovy, S.R., Burton, M.G., Erickson, E.F., et al., 1998 ApJL, 492, 151

Tofani, G., Felli, M., Taylor, G.B., \& Hunter, T.R., 1995 A $\& A S, 112,299$

Torrelles, J.M., Verdes-Montenegro, L., Ho, P.T.P., Rodríguez, L.F., \& Canto, J. 1993 ApJ, 410, 202

Wood, D.O.S., \& Churchwell, E., 1989 ApJS, 69, 831

Wright, M.C.H., Plambeck, R.L., Mundy, L.G., \& Looney, L. W., 1995 ApJL, 455, 185

Zhang, Q., Hunter, T.R., \& Sridharan, T.K., 1998 ApJL, 505, 151

Zheng X.W., Ho, P.T.P, Reid, M.J., \& Schneps, M.H., 1985 ApJ, 293, 522 